\title{
THE ABSOlute PROPER MOTION OF THE PLEIADES ClUSTER
}

\author{
W. F. van Altena and B. F. Jones \\ Yerkes Observatory
}

The establishment of a truly inertial reference system is a problem that has defied solution for many years. However, with the completion of the Lick proper motion survey (Wright 1950) and the USSR program (Deutsch $1954)$ the situation for the northern hemisphere should be satisfactory.

Ideally one wishes to determine the motions of the fundamental stars directly with respect to the galaxies, but this is not practical due to the faintness of the galaxies and the brightness of the fundamental stars. To bridge the gap a set of intermediate brightness stars are used and in the process absolute proper motions are obtained for these stars also.

It is the purpose of this paper to report on a project to determine the absolute proper motion of the bright fundamental stars in the Pleiades cluster directly with respect to the galaxies and then to determine corrections to the fundamental catalogues for this area of the sky. This is made possible by the large range in brightness of the stars in the Pleiades cluster, from 3rd - 20th magnitude, and by the presence of galaxies near the Pleiades. The faint Pleiades members used are the white dwarfs discovered by Luyten and Herbig (1960) and the list of flare stars by Haro (1968) and Rosino (1966). The survey for suitable galaxies for measurement was made by Mr. E. Vilkki on a glass copy of the Palomar Observatory Sky Survey and then these objects were located on plates taken with the twenty-inch Carnegie Astrograph of the Lick Observatory. About 100 suitable galaxies have been found in forty square degrees around the Pleiades. The mean magnitude and color of these galaxies is approximately equal to that of the faint flare stars in the Pleiades. Preliminary measurements have shown that the unit weight mean error for the galaxy plate constant solutions is about $\pm 4.0 \mu$, while for the stars it is about $\pm 3.0 \mu$. With an epoch difference of 16 years, the expected accuracy of the absolute proper motion is $\pm 0: 002 / \mathrm{yr}(\mathrm{m} . \mathrm{e}$.$) .$

An estimate of the accuracy with which the fundamental system represents an inertial system may be obtained by comparing individual catalogues with the fundamental system. For the Pleiades region of the sky deviations as large as 0:'15 in each coordinate occur for an individual catalogue (Scott 1963). One hopes that the average results obtained from a number of different telescopes will be systematically more correct than the individual catalogue. This hope must be tempered however with the realization that most transit instruments are of a similar design and may be effected by similar errors which would not average out. If similar deviations also occur for the early catalogues then we are led to the conclusion that the system of proper motions may be in error by $\pm 0 ! n 004 / y r$. Although our expected accuracy is only a factor of two smaller than the expected error, we hope to be able to set an upper limit on the error of the fundamental system in the region of the Pleiades cluster. 


\section{ACKNOWLEDGEMENTS}

We would like to thank Dr. S. Vasilevskis for the use of the twentyinch Carnegie astrograph plates and for providing time on the automatic measuring machine, and Dr. W. W. Morgan for helpful discussions.

This research has been supported in part by the Office of Naval Research under contract N00014-67-A-0285-0004 and by the National Science Foundation under grant NSF GP13771.

\section{RE FERENCES}

Deutsch, A. N. 1954, Trans. I. A. U. , 8, 789.

Haro, G. 1968, Flare Stars in Nebulae and Interstellar Matter,

Ed. B. M. Middlehurst and L.H. Aller, University of Chicago Press, Chicago, P. 141.

Luyten, W.J. and Herbig, G.H. 1960, Harvard Announcement Card No. 1474. Rosino, L. 1966, Memorie Soc. Astron. Italliana, 37, 717.

Scott, F. P. 1963, The System of Fundamental Proper Motions in Basic Astronomical Data, Ed. K. Aa. Strand, Univ. of Chicago Press, Chicago, P. 11.

Wright, W. H. 1950, Proc. Amer. Phil. Soc., 94, 1. 